\title{
A chromosome-level genome assembly of rugged rose (Rosa rugosa) provides insights into its evolution, ecology, and floral characteristics
}

\author{
Fei Chen'1, Liyao Su', Shuaiya Hu', Jia-Yu Xue ${ }^{1,2}$, Hui Liu', Guanhua Liu', Yifan Jiang', Jianke Du', Yushan Qiao (D)', \\ Yannan Fan $\mathbb{1}^{3,4}$, Huan Liu $\mathbb{1}^{3,4}$, Qi Yang ${ }^{5}$, Wenjie Luㄴ, Zhu-Qing Shao ${ }^{6}$, Jian Zhang ${ }^{7}$, Liangsheng Zhang ${ }^{8}$, \\ Feng Chen ${ }^{9}$ and Zong-Ming (Max) Cheng ${ }^{1}$
}

\begin{abstract}
Rosa rugosa, commonly known as rugged rose, is a perennial ornamental shrub. It produces beautiful flowers with a mild fragrance and colorful seed pods. Unlike many other cultivated roses, $R$. rugosa adapts to a wide range of habitat types and harsh environmental conditions such as salinity, alkaline, shade, drought, high humidity, and frigid temperatures. Here, we produced and analyzed a high-quality genome sequence for $R$. rugosa to understand its ecology, floral characteristics and evolution. PacBio HiFi reads were initially used to construct the draft genome of R. rugosa, and then $\mathrm{Hi}-\mathrm{C}$ sequencing was applied to assemble the contigs into 7 chromosomes. We obtained a 382.6 Mb genome encoding 39,704 protein-coding genes. The genome of R. rugosa appears to be conserved with no additional whole-genome duplication after the gamma whole-genome triplication (WGT), which occurred 100 million years ago in the ancestor of core eudicots. Based on a comparative analysis of the high-quality genome assembly of $R$. rugosa and other high-quality Rosaceae genomes, we found a unique large inverted segment in the Chinese rose $R$. chinensis and a retroposition in strawberry caused by post-WGT events. We also found that floral development- and stress response signaling-related gene modules were retained after the WGT. Two MADS-box genes involved in floral development and the stress-related transcription factors DREB2A-INTERACTING PROTEIN 2 (DRIP2) and PEPTIDE TRANSPORTER 3 (PTR3) were found to be positively selected in evolution, which may have contributed to the unique ability of this plant to adapt to harsh environments. In summary, the high-quality genome sequence of $R$. rugosa provides a map for genetic studies and molecular breeding of this plant and enables comparative genomic studies of Rosa in the near future.
\end{abstract}

\section{Introductions}

Rosa rugosa is a perennial shrub tree that grows to 1-1.5 $\mathrm{m}$ tall and is native to Eastern Asia. It blooms and produces edible hips (the seed pods) in summer and early autumn. $R$. rugosa has been utilized in many ways. Because of its attractive pink flowers, $R$. rugosa is often

Correspondence: Zong-Ming (Max) Cheng (zcheng@utk.edu)

${ }^{1}$ College of Horticulture, Nanjing Agricultural University, Nanjing 210095, China

${ }^{2}$ Academy for Advanced Interdisciplinary Studies, Nanjing Agricultural

University, Nanjing 210095, China

Full list of author information is available at the end of the article

These authors contributed equally: F. Chen, L. Su used to create windbreaks and hedges. It has also been cultivated in North America and Europe as an introduced ornamental plant. The fruits of $R$. rugosa possess antioxidant activity and antibacterial activity due to their high contents of phenolic and flavonoid compounds and ascorbic acid ${ }^{1,2}$. It is able to control soil erosion and is planted along highways in Germany and Denmark ${ }^{3}$. Because of the high level of biosynthesis of pleasant volatile compounds in its flowers, $R$. rugosa has been used as an important source for the production of essential oil ${ }^{4}$. In breeding, $R$. rugosa has been widely used for breeding salt-resistant Rosa varieties. Although $R$. rugosa has many

\section{(c) The Author(s) 2021}

(c) (i) Open Access This article is licensed under a Creative Commons Attribution 4.0 International License, which permits use, sharing, adaptation, distribution and reproduction cc) in any medium or format, as long as you give appropriate credit to the original author(s) and the source, provide a link to the Creative Commons license, and indicate if changes were made. The images or other third party material in this article are included in the article's Creative Commons license, unless indicated otherwise in a credit line to the material. If material is not included in the article's Creative Commons license and your intended use is not permitted by statutory regulation or exceeds the permitted use, you will need to obtain permission directly from the copyright holder. To view a copy of this license, visit http://creativecommons.org/licenses/by/4.0/. 
advantages, research on its molecular breeding and domestication has not even begun, partly due to the lack of high-quality genome sequences.

Also known as rugged rose, $R$. rugosa can adapt to many environmental conditions, such as salinity and alkaline soils, shade, frigid temperatures, drought, and high humidity. These excellent abilities make $R$. rugosa ideal for gene mining and molecular breeding to produce novel Rosa varieties. In some places, $R$. rugosa has become invasive ${ }^{5}$, attesting to its ability to adapt to new environments. However, the molecular mechanisms underlying this adaptability are largely unknown.

Following the rapid development of genome sequencing technologies and bioinformatic technologies, hundreds of angiosperm genomes have been reported ${ }^{6-8}$. The Rosa genus includes $\sim 200$ species with quite different morphological traits ${ }^{9}$. Within the Rosa genus, the first draft genome sequence of wild Rosa multiflora was released in $2018^{10}$. Since then, two chromosome-level genomes of Rosa chinensis, also known as Chinese rose, have been released $^{11,12}$. For $R$. rugosa, only the chloroplast genome ${ }^{13}$ and mitochondrial genome ${ }^{14}$ have been reported. A highquality genome sequence for $R$. rugosa would not only enable comparative genomic studies of Rosa species but also reveal the mechanisms underlying its ornamental traits, such as floral biology and its unique ecology.

Here, we report the first chromosome-level genome assembly of $R$. rugosa, relying on HiFi sequencing and $\mathrm{Hi}-\mathrm{C}$ scaffolding technology. Based on this high-quality genome assembly, we studied the genomic structural differences between $R$. rugosa and $R$. chinensis. We also revealed the genetics responsible for floral biology. The mechanisms that account for its evolution and

Table 1 Statistics of the $R$. rugosa genome assembly and annotation

\begin{tabular}{ll}
\hline Feature & Value \\
\hline Raw data of PacBio-HiFi sequencing (Gb) & 59.24 \\
Raw data of 10X Genomics (Gb) & 80.91 \\
Raw data of Hi-C sequencing (Gb) & 150.6 \\
Estimated genome size (Mb) & 454.78 \\
Assembled contigs (Mb) & 382.64 \\
Contig N50 (Mb) & 15.36 \\
Number of contig & 105 \\
Largest contig (Mb) & 31.80 \\
Total size of chromosome (Mb) & 375.79 \\
GC content (\%) & 39.30 \\
Heterozygosity (\%) & 0.71 \\
Number of genes & 39,704 \\
\hline
\end{tabular}

adaptation to harsh environments were explored here as well.

\section{Results and discussion}

Genome sequencing and assembly

We used a combination of sequencing technologies, including PacBio-CCS (HiFi), 10X genomics, and $\mathrm{Hi}-\mathrm{C}$, to construct the reference genome for $R$. rugosa. We obtained a total of $59.24 \mathrm{~Gb}$ HiFi clean data and $80.91 \mathrm{~Gb}$ 10X genomics clean data, respectively. We employed $K$-mer-based statistics to predict genome size, and it was estimated to be $454.78 \mathrm{Mb}$. The assembled genome is $382.64 \mathrm{Mb}$ with a contig N50 of $15.36 \mathrm{Mb}$ (Table 1), significantly longer than that in $R$. chinensis (contig N50= $3.4 \mathrm{Mb})^{12}$ or woodland strawberry Fragaria vesca (contig $\mathrm{N} 50=7.9 \mathrm{Mb})^{15}$. The GC content of the $R$. rugosa genome was $39.30 \%$ (Table 1), which was very similar to that of F. vesca (38.98\%) and R. chinensis (38.84\%). To assemble the contigs into chromosomes, we applied Hi-C sequencing technology and anchored $98.21 \%$ of the sequences onto 7 chromosomes (Fig. 1, Supplementary Table 1). Based on this high-quality genome assembly, we evaluated the genome completeness of $R$. rugosa using BUCSO with the embryophyte_odb10 database. The genome assembly completeness reached $93.2 \%$, and the

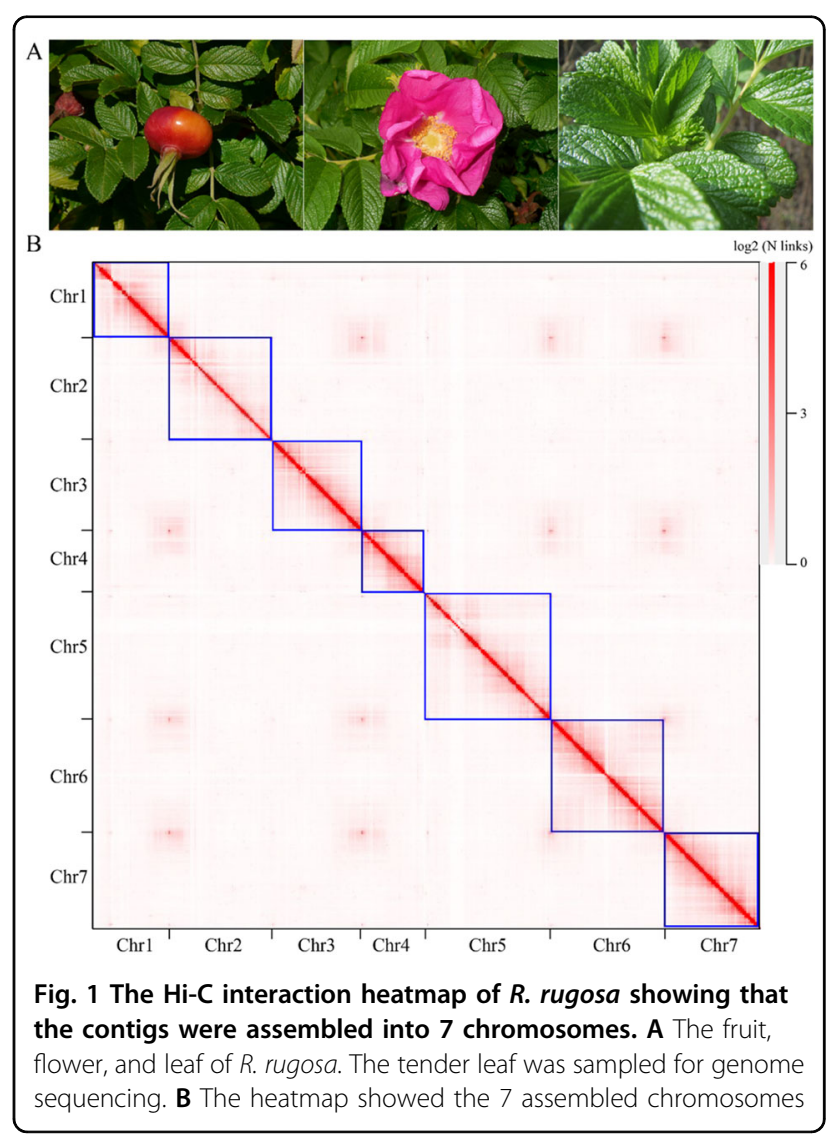


Table 2 Repeat sequences in the R. rugosa genome

\begin{tabular}{|c|c|c|c|c|}
\hline & Type & Number of elements & Length occupied (bp) & Percentage of sequence (\%) \\
\hline \multirow[t]{11}{*}{ Retroelements } & & 111,329 & 118367,513 & 30.04 \\
\hline & SINEs: & 5594 & 793,802 & 0.2 \\
\hline & Penelope & 30 & 19,393 & 0 \\
\hline & LINEs: & 20,751 & $12,160,801$ & 3.09 \\
\hline & L2/CR1/Rex & 457 & 449,390 & 0.11 \\
\hline & L1/CIN4 & 20,036 & $11,601,916$ & 2.94 \\
\hline & LTR elements: & 84,984 & $105,412,910$ & 26.75 \\
\hline & BEL/Pao & 53 & 14,816 & 0 \\
\hline & Ty1/Copia & 32,581 & $38,364,733$ & 9.74 \\
\hline & Gypsy/DIRS1 & 50,138 & $65,699,666$ & 16.67 \\
\hline & Retroviral & 255 & 74,660 & 0.02 \\
\hline \multirow[t]{4}{*}{ DNA transposons } & & 83,506 & $25,795,514$ & 6.55 \\
\hline & hobo-Activator & 21,137 & $6,286,361$ & 1.6 \\
\hline & Tc1-IS630-Pogo & 204 & 46,086 & 0.01 \\
\hline & PiggyBac & 376 & 130,940 & 0.03 \\
\hline Rolling-circles & & 4111 & $2,498,640$ & 0.63 \\
\hline Unclassified: & & 172,477 & $46,290,831$ & 11.75 \\
\hline Total repeats: & & & $190,453,858$ & 48.33 \\
\hline Small RNA: & & 5982 & 926,674 & 0.24 \\
\hline Satellites: & & 875 & 279,368 & 0.07 \\
\hline Simple repeats: & & 103,313 & $3,857,918$ & 0.98 \\
\hline Low complexity: & & 16,875 & 814,967 & 0.21 \\
\hline
\end{tabular}

gene prediction completeness reached $94.4 \%$. We further compared the completeness of $R$. rugosa with the released Rosaceae genomes of $R$. chinensis, strawberry (F. vesca), peach $(P$. persica), apple $(M$. domestica) and pear (P. bretschneideri). Their proportions were similar to those of $R$. rugosa (Supplementary Table 2), indicating the high quality of our genome assembly.

\section{Genome components}

The $R$. rugosa genome was composed of $50.27 \%$ repetitive sequences (Table 2). Most of these repetitive sequences are long terminal repeats (LTRs), including Gypsy and Copia, accounting for $26.75 \%$ of the total genome. The proportion of LTRs in R. rugosa was much greater than that in Fragaria spp. such as $F$. vesca $(\sim 16 \%)^{16}$ and $F$. nilgerrensis $(16.5 \%)^{17}$ but slightly less than that in $R$. chinensis $(28.3 \%)^{12}$, suggesting the rapid evolution of LTRs in Rosaceae plants. The $R$. rugosa genome encodes 39,704 protein-coding genes, close to the number in $R$. chinensis ${ }^{12}$. Moreover, we annotated 37.32\%, $87.58 \%$, and $23.03 \%$ of genes using the Gene Ontology
(GO), Kyoto Encyclopedia of Genes and Genomes (KEGG) and Clusters of Orthologous Groups (COG) databases (Supplementary Figs. 1, 2, 3). We mapped the genes and repetitive elements to the 7 chromosomes (Fig. 2).

\section{Evolution of the $R$. rugosa genome}

To study the evolution of the $R$. rugosa genome, we constructed a species tree of $R$. rugosa and representative Rosaceae species using phylogenomics. We obtained 321 high-confidence single-copy nuclear genes across 8 eudicot species. $R$. rugosa is closely related to $R$. chinensis, diverging $\sim 5.26$ million years ago (Fig. 3). Although they are close relatives in the Rosa genus, the gene orthogroups differ greatly in these two species, gaining 5418 and 1764 in $R$. chinensis and R. rugosa, respectively, and losing 2404 and 4676 in $R$. chinensis and $R$. rugosa, respectively. The genus Rosa could be divided into two groups: group I: Pimpinellifoliae+Rosa+Carolinae and group II: Gallicanae + Synstylae + Chinenses +Laevigatae + Caninae +Banksianae +Microphyllae+Bracteatae. This significant orthogroup 


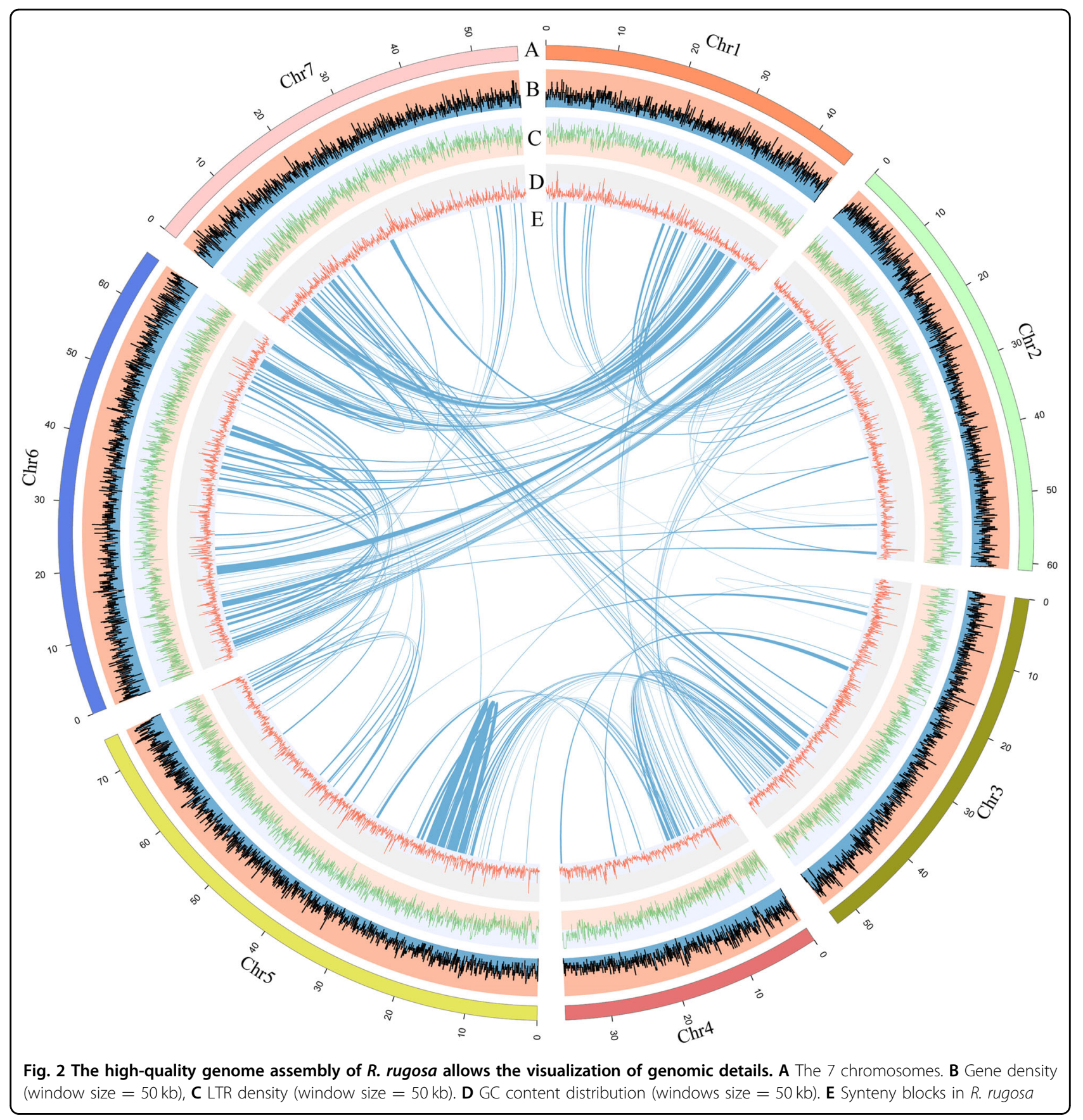

difference may be because $R$. rugosa belongs to Group I and $R$. chinensis belongs to Group $\mathrm{II}^{18}$.

We then explored the orthogroup variations between $R$. rugosa and $R$. chinensis. We studied both the contracted and expanded orthogroups in these two species (Table 3). We showed that $R$. rugosa lost several orthogroups, including OG0000650 (aldolase superfamily), OG0000325 (aminotransferase-like), OG0001051 (IBR domain-containing), OG0000709 (NB-ARC domaincontaining disease resistance), and OG0000761 (NB-ARC domain-containing disease resistance), but had more NBARC domain-containing disease resistance protein genes than OG0000869.

The publications of hundreds of angiosperm genomes ${ }^{6}$ has revealed that polyploidization events have occurred frequently, with at least four waves ${ }^{19}$, contributing to the genomic materials for innovation ${ }^{20}$. We calculated the gene $K \mathrm{~s}$ values in $R$. rugosa, $R$. chinensis, and Vitis vinifera. We found that their shared feature is a single peak at 1.31.5 (Fig. 4A-C). We then compared the whole-genome 


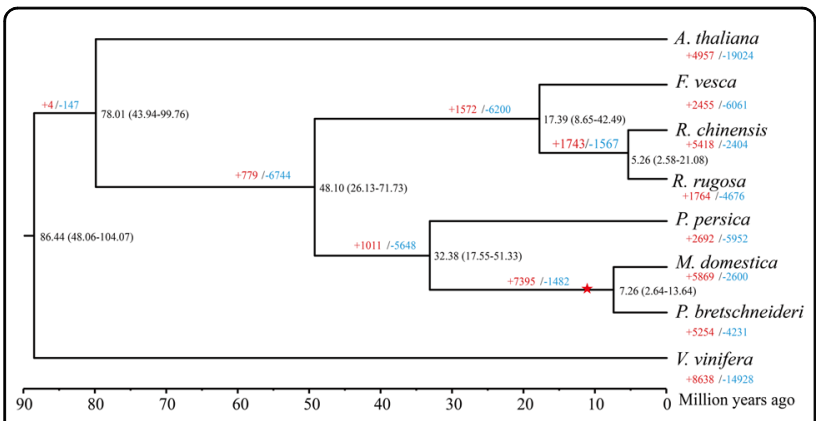

Fig. 3 A phylogenomic species tree of $R$. rugosa and eight other representative species. This tree was constructed using

321 stringent single-copy nuclear protein-coding genes, showing gene family contraction and expansion. The numbers above the branches represent the number of gene families with either expansion (red) or contraction (blue). The numbers at the node indicate divergence time and 95\% confidence interval. The species used in the tree are Arabidopsis thaliana, Fragaria vesca, Rosa chinensis, Rosa rugosa, Prunus persica, Malus domestica, and Pyrus bretschneideri. The red star indicates the whole-genome duplication in the ancestor of apple (M. domestica) and pear (P. bretschneideri)

syntenic patterns and still did not find any recent WGD. These results show that the Rosa species experienced only the eudicot-specific WGT event, similar to grapes ${ }^{21}$. This result is consistent with previous reports for other Rosa species $^{22}$.

We compared the syntenic patterns of $R$. rugosa with those of other representative species (Fig. 4D). We showed that $R$. rugosa has very conserved syntenic relationships with grape. For example, VvChr1 and VvCh5 correspond to RrChr1, VvChr9, and VvChr11, and half of VvChr14 matches RrChr6 (Fig. 4D, Supplementary Fig. 4). In the genomes of $R$. rugosa and $R$. chinensis, every chromosome matched each other well. However, when compared with two other Rosaceae species, namely, peach (P. persica) and wild strawberries (F. vesca), we found that a large segment composed of $10.44 \mathrm{Mb}$ of chromosome was reversed in $R$. chinensis but in the exact same order in other species (Fig. 4D, Supplementary Fig. 5). In addition, we found that a segment $1.56 \mathrm{Mb}$ in length was translocated in $F$. vesca. These results suggest that genomes within the Rosa genus are very conserved in terms of synteny and that small genetic changes could contribute to morphological variations.

Since we did not find significant expansion or loss of genes related to the salt stress response or water stress in $R$. rugosa compared to $R$. chinensis (Table 3), we then investigated the contribution of WGT to $R$. rugosa.

We studied the WGT and its contribution to floral evolution in $R$. rugosa and $R$. chinensis. $R$. rugosa has large, pink, and fragrant flowers. We analyzed the genes retained after WGT to determine whether floral genes could have expanded after this ancient polyploidization event. Gene Ontology annotation of all $R$. rugosa proteincoding genes showed that 146 genes, compared to 67 genes in $R$. chinensis, were involved in floral organ development (Fig. 5A, Supplementary Figs. 6, 7), suggesting that $R$. rugosa retained many more genes for floral-related traits. Floral organ development was divided into four categories, including floral organ development, floral whorl development, floral organ morphogenesis and floral organ formation, according to the agriGO analyses. Among them, 34 genes, including kinase proteins (LRR kinases) and transcription factors (KNOX/ELK, MYB, zinc finger and MADS-box), were involved in all four aspects in R. rugosa (Fig. 5B). However, only 13 genes were involved in all four aspects in R. chinensis (Supplementary Table 3). Then, we compared the floral organ determination genes and the MADS-box genes in $R$. rugosa, $R$. chinensis, and A. thaliana. We found a total of 92 MADS-box genes in $R$. rugosa, slightly more than that in $R$. chinensis (84 MADS-box genes) (Supplementary Fig. 8). The $S$-locus of $R$. rugosa was investigated for the first time and compared with other Rosaceae species (Supplementary Fig. 9). The results showed that there were $19 F$-box genes and one $S$-RNase gene in $R$, rugosa. Unlike Prunus spp., R. rugosa's S-locus size was similar to that in Maleae spp., suggesting that the self-incompatibility recognition mechanism was closer to or belonged to the multifactor recognition model.

R. rugosa plants are economically important partly due to the high essential oil production of their flowers. Monoterpenes are the main constituents of essential oils, accounting for $50-70 \%$ of the total content ${ }^{23,24}$. Due to the lack of genome sequences, only a fraction of genes could be identified using transcriptomes or comparative genomic studies ${ }^{24}$. Here, a total of 53 terpene synthases (TPSs), which are key genes responsible for terpene biosynthesis, were identified from the genome of $R$. rugosa (Supplementary Fig. 10). The RrTPSs were distributed into five subfamilies (TPS-a, b, c, g and e/f) based on clustering with TPS identified from model angiosperm species ${ }^{25}$. Eighteen and four RrTPS genes were found to belong to the TPS-g and TPS-b subfamilies, respectively. Because TPS- $g$ and TPS-b are mainly involved in monoterpene biosynthesis, these 22 RrTPS genes are the main candidates responsible for the high-level production of monoterpenes in essential oil. Twenty-six RrTPS genes were identified to be members of the TPS-a subfamily with putative sesquiterpene synthase functions. In addition, the TPS family in $R$. rugosa contains two members in the TPSc subfamily and 3 members in the TPS-e/f subfamily. Further phylogenetic analysis indicated that each RrTPS gene, a member of TPS- $g$, has corresponding orthologs in the genome of $R$. chinensis (Supplementary Fig. 10), suggesting a close evolutionary relationship between the two TPS families from $R$. rugosa and $R$. chinensis. 
Table 3 The expansion and contraction of orthogroups between $R$. rugosa and $R$. chinensis

\begin{tabular}{|c|c|c|c|c|}
\hline Family & R. rugosa & R. chinensis & Expansion or contraction & Annotation \\
\hline OG0000000 & 338 & 7 & 331 & Ribonuclease H-like superfamily \\
\hline OG0000172 & 60 & 0 & 60 & Retroviridae gag-proteins \\
\hline OG0000013 & 80 & 24 & 56 & Ribonuclease H-like superfamily \\
\hline OG0000189 & 55 & 1 & 54 & Retroviridae gag-proteins \\
\hline OG0000016 & 74 & 20 & 54 & Ribonuclease H-like superfamily \\
\hline OG0000055 & 69 & 18 & 51 & Ribonuclease H-like superfamily \\
\hline OG0000044 & 63 & 14 & 49 & DNA/RNA polymerases superfamily protein \\
\hline OG0000180 & 48 & 9 & 39 & DNAse I-like superfamily protein \\
\hline OG0000250 & 42 & 4 & 38 & Cysteine-rich receptor-like protein kinase \\
\hline OG0000516 & 39 & 1 & 38 & Zinc knuckle (CCHC-type) family protein \\
\hline OG0000177 & 47 & 10 & 37 & Ribonuclease H-like superfamily \\
\hline OG0000564 & 37 & 0 & 37 & Ribonuclease H-like superfamily \\
\hline OG0000174 & 43 & 7 & 36 & Cysteine-rich receptor-like protein Kinase \\
\hline OG0000517 & 38 & 2 & 36 & Ribonuclease H-like superfamily \\
\hline OG0000126 & 40 & 5 & 35 & Cysteine-rich receptor-like protein kinase \\
\hline OG0000098 & 45 & 10 & 35 & DNA/RNA polymerases Superfamily protein \\
\hline OG0000652 & 36 & 1 & 35 & Ribonuclease H-like superfamily \\
\hline OG0000384 & 35 & 1 & 34 & Cysteine-rich RECEPTOR-like kinase \\
\hline OG0000028 & 59 & 25 & 34 & MuDR family transposase \\
\hline OG0000052 & 44 & 10 & 34 & Ribonuclease H-like superfamily \\
\hline OG0000116 & 46 & 14 & 32 & WUS/WUSCHEL \\
\hline OG0000869 & 32 & 1 & 31 & NB-ARC domain-containing Disease resistance protein \\
\hline OG0000100 & 36 & 5 & 31 & Ribonuclease H-like superfamily \\
\hline OG0000514 & 35 & 4 & 31 & Ribonuclease H-like superfamily \\
\hline OG0000288 & 32 & 3 & 29 & zinc knuckle (CCHC-type) family protein \\
\hline OG0001051 & 0 & 31 & -31 & IBR domain-containing protein \\
\hline OG0000450 & 3 & 35 & -32 & NB-ARC domain-containing Disease resistance protein \\
\hline OG0000020 & 22 & 54 & -32 & TIR-NBS-LRR class \\
\hline OG0000762 & 1 & 34 & -33 & NB-ARC domain-containing Disease resistance protein \\
\hline OG0000761 & 0 & 35 & -35 & NB-ARC domain-containing Disease resistance protein \\
\hline OG0000049 & 31 & 66 & -35 & TIR-NBS-LRR class \\
\hline OG0000709 & 0 & 36 & -36 & NB-ARC domain-containing Disease resistance protein \\
\hline OG0000650 & 0 & 37 & -37 & Aldolase superfamily protein \\
\hline OG0000041 & 21 & 63 & -42 & Nuclease \\
\hline OG0000147 & 1 & 47 & -46 & ANTHRANILATE SYNTHASE BETA SUBUNIT 1 \\
\hline OG0000325 & 0 & 48 & -48 & Aminotransferase-like, plant mobile domain family protein \\
\hline OG0000009 & 13 & 66 & -53 & Leucine-rich repeat (LRR) family \\
\hline
\end{tabular}




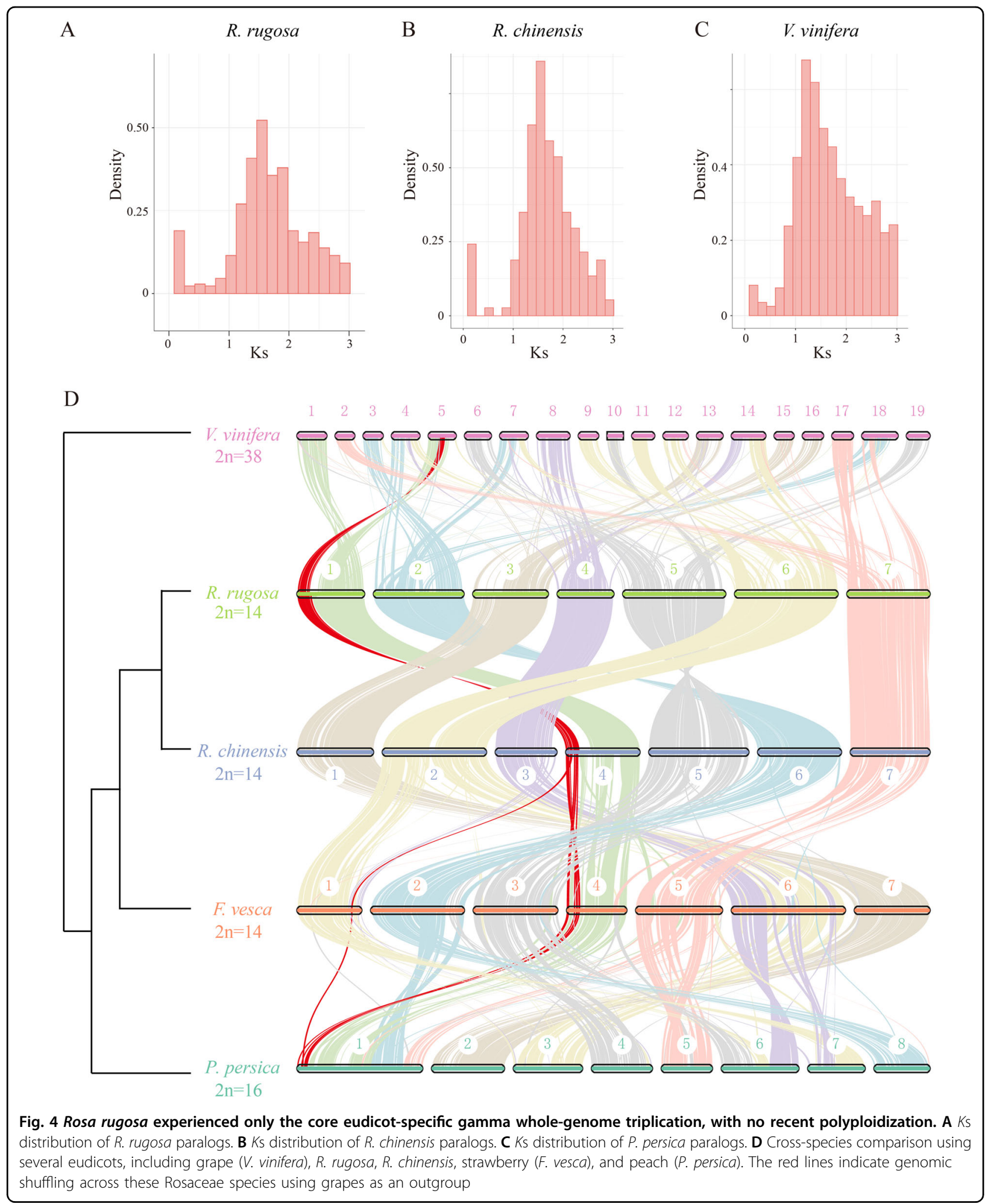

$R$. rugosa can adapt to drought, salinity, and alkaline soils and can even become invasive in some places ${ }^{3}$. However, $R$. chinensis does not have such abilities. By pathway enrichment of all $R$. rugosa genes (Supplementary Fig. 2), we showed that 850 genes in $R$. rugosa were involved in environmental adaptation. To trace the origin 


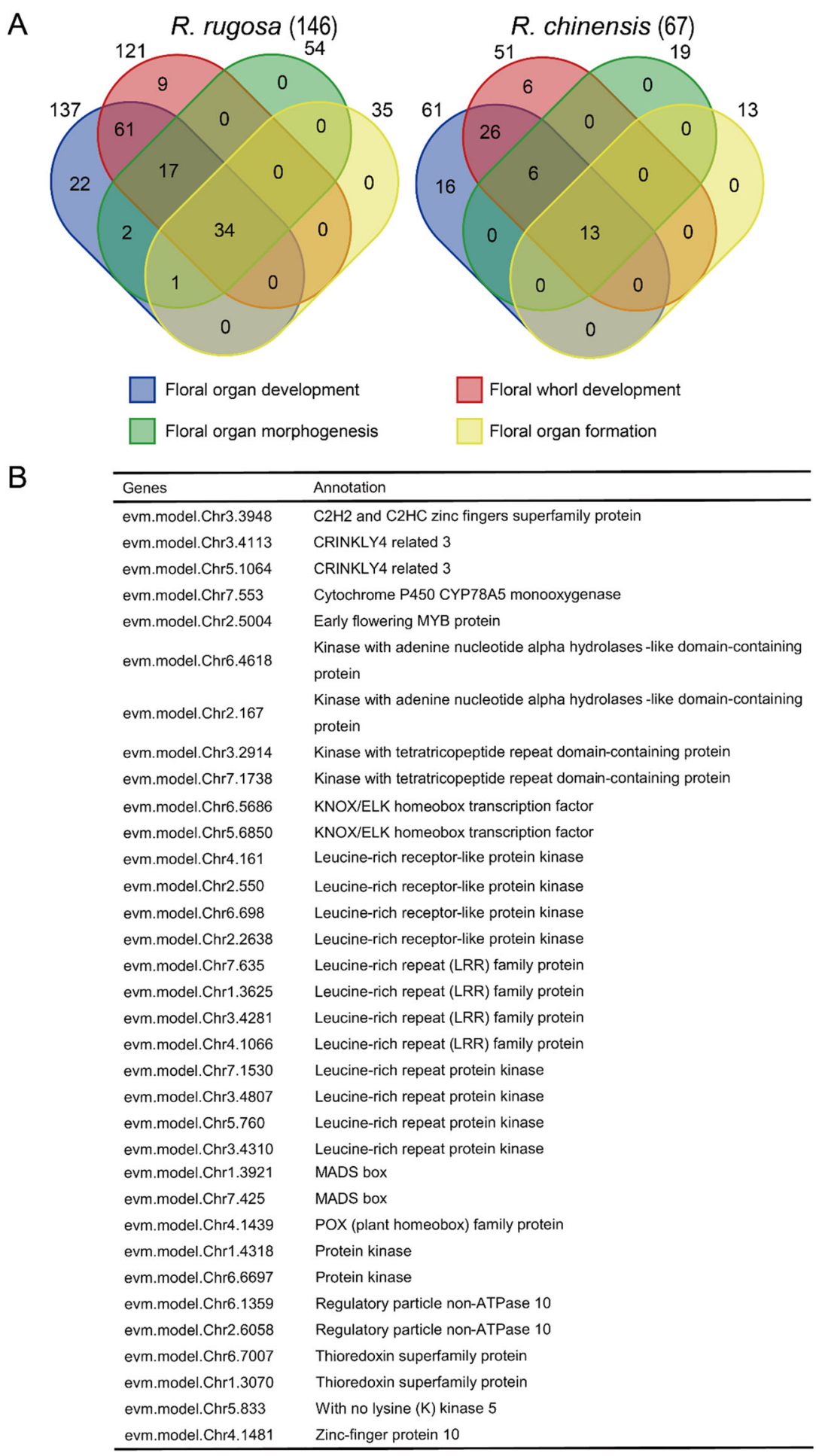

Fig. 5 The floral developmental genes were retained after gamma WGT in $\boldsymbol{R}$. rugosa. A The Venn diagram shows the distribution of genes involved in floral organ development, floral whorl development, floral organ morphogenesis, and floral organ formation from $R$. chinensis and R. rugosa. B Annotation of the 34 genes involved in four aspects of floral development in $R$. rugosa identified a series of kinase and transcription factor genes 
and evolution of these stress-related genes, we found that two pathways, salt stress and water stress (water deprivation or drought), were significantly retained and enriched after WGT (Fig. 6A for R. rugosa, Supplementary Fig. 11 for $R$. chinensis). In each module of $R$. rugosa, the number of genes was significantly higher than that in $R$. chinensis. Furthermore, we constructed a Venn diagram (Fig. 6B, C) to show the genes that might be involved in cross talk related to these abiotic stresses. Eventually, we found 11 and 7 genes in $R$. rugosa and $R$. chinensis that were predicted to be involved in these four abiotic stress responses, respectively (Supplementary Table 4, Supplementary Table 5). Notably, among these module genes, we found that two paralogs of DREB2A-INTERACTING PROTEIN 2 (DRIP2) in $R$. rugosa had been subjected to positive selection pressure (Fig. 6D, Supplementary Table 6). Furthermore, we found two drought/water stressrelated DRIP2 genes in $R$. rugosa but only one in $R$. chinensis or Arabidopsis, with potential gene neofunctions in $R$. rugosa's adaptation to stressful environments. Meanwhile, we found that the number of PTR3 genes, which encode dipeptide and tripeptide transporters involved in responses to high $\mathrm{NaCl}$ concentrations, expanded to 7 in $R$. rugosa but only four in $R$. chinensis, 5 in $F$. vesca, 3 in $P$. persica and 3 in A. thaliana. Two PTR3 (PEPTIDE TRANSPORTER 3) genes under positive selection pressure were detected (Fig. 6E, Supplementary Table 6). Therefore, these genes might provide $R$. rugosa with its unique ability to adapt to high salinity environments and water stresses.

Finally, as shown in Fig. 7, we constructed the salt stress response pathway of $R$. rugosa. Meanwhile, we compared the differences in the number of genes between A. thaliana, $R$. rugosa and $R$. chinensis (Supplementary Table 7). There was no difference in the number of genes among these sampled species, suggesting that $R$. rugosa did not cope with salt stress using gene dosage, but rather using transcription-, translation-, or metabolome-level regulation.

\section{Conclusions}

As a popular ornamental plant, R. rugosa is widely cultivated. The flowers of $R$. rugosa have been utilized for essential oil production and dried to produce flower tea. The economic value of this plant will certainly grow if molecular breeding accelerates the production of novel cultivars with optimized essential oil content and improved floral traits. A high-quality reference genome will provide a map for the identification of genes responsible for key agronomic traits and provide insights into how rugosa rose evolved during its long evolutionary history. This study provides for the first time the valuable resource of a $R$. rugosa genome for the rose research community. Through analysis of the genome sequence of $R$. rugosa and comparative genomic analyses, we provide novel insights into the biology, ecology and evolution of $R$. rugosa from three main perspectives. From the perspective of structural genomics, we show a large reversed segment in $R$. chinensis and a translocation in strawberry. From the perspective of floral biology, we found that more MADS-box genes were retained in $R$. rugosa than in $R$. chinensis, suggesting their potential roles in floral development in $R$. rugosa. From the perspective of stress biology, a number of stress-related genes were found to be specifically expanded and retained in $R$. rugosa, potentially contributing to its adaptation to stressful environments.

\section{Materials and methods \\ Plant samples and DNA/RNA extraction}

The $R$. rugosa plants were sampled from Nanjing Agricultural University. For genome sequencing, we collected mature healthy $R$. rugosa leaves. For transcriptome sequencing, the roots, stems, and leaves of $R$. rugosa were obtained. All samples were quickly frozen in liquid nitrogen and stored in a $-80^{\circ} \mathrm{C}$ freezer. We used a QIAGEN® Genomic DNA extraction kit (Cat\#13323, QIAGEN) to extract genomic DNA according to the standard operating procedure provided by the manufacturer. We isolated total RNA for RNA sequencing by TRIzol reagent according to the manufacturer's instructions.

\section{Sequencing and library construction}

We used a total of $15 \mu \mathrm{g}$ genomic DNA to construct a SMRTbell target size library for PacBio-HiFi sequencing according to a standard protocol. We sheared genomic DNA to the expected size of fragments for sequencing on a PacBio Sequel II instrument with Sequencing Primer V2 and Sequel II Binding Kit 2.0 in Grandomics. To construct the Hi-C library, we digested cross-linked chromatin into units with Dpn II, marked by incubation with biotin-14-dCTP and ligated the units by biotinylation. Finally, the ligated genomic DNA was sheared to $100 \mathrm{bp}$ by StLFT technology and sequenced using the DIPSEQ platform at BGI, China. One microgram of sample RNA was used to build an RNA library with a TruSeq RNA Library Preparation Kit (Illumina, USA) following the manufacturer's recommendations.

\section{Genome assembly and quality evaluation}

Approximately $59.2 \mathrm{~Gb}$ of raw $\mathrm{HiFi}$ sequencing reads was obtained from the rosa DNA library. We first used HiCanu v2.2.1 ${ }^{26}$ for preliminary assembly of the rosa genome. Then, Redundans v $0.14 \mathrm{a}^{27}$ was performed to remove the redundant sequences. A total of $150.6 \mathrm{~Gb}$ of $\mathrm{Hi}-\mathrm{C}$ data were obtained to anchor the contig onto the chromosome. We aligned $\mathrm{Hi}-\mathrm{C}$ reads to assembly by BWA v 0.7.17-r1188 ${ }^{28}$. Next, the draft assembly genome was scaffolded with Hi-C reads by 3D-DNA v180114 ${ }^{29}$. 

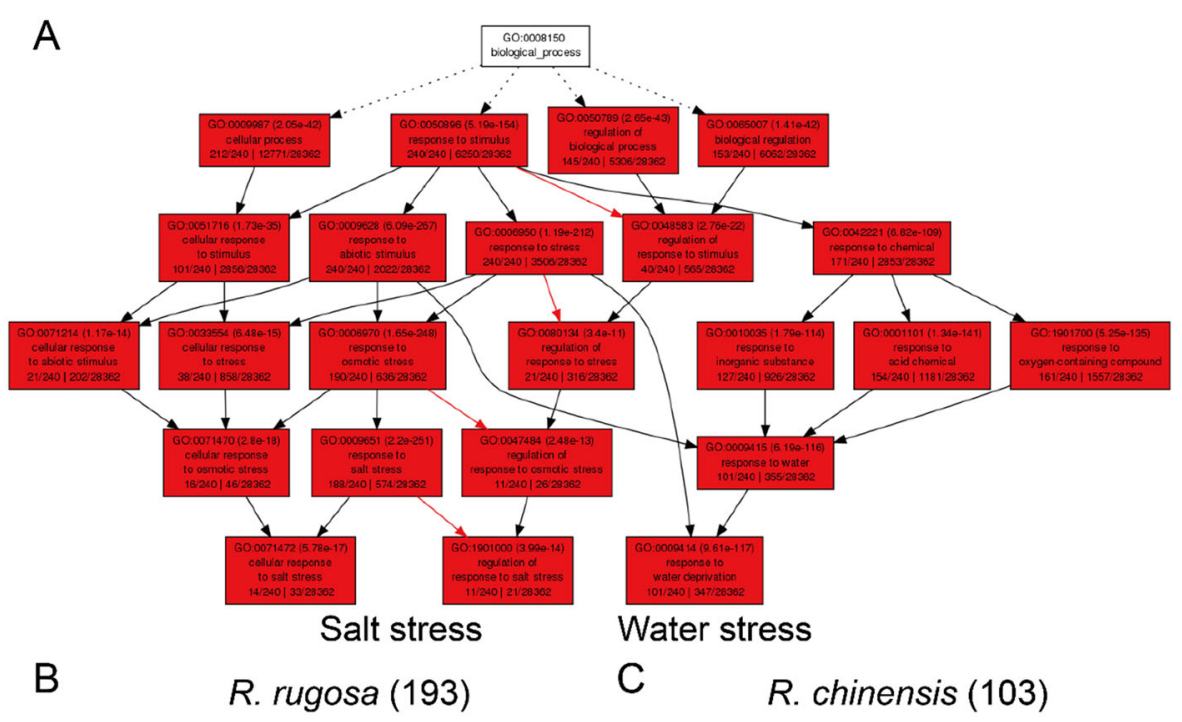

C R. chinensis (103)
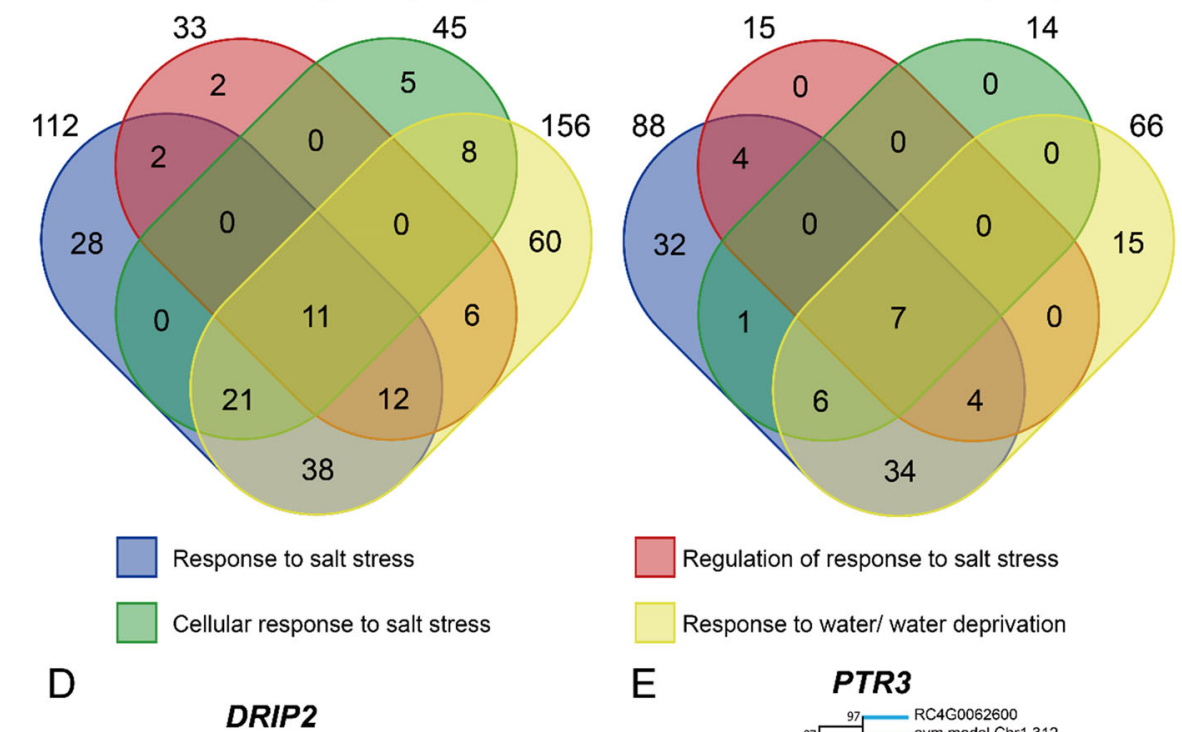

$\mathrm{E}$
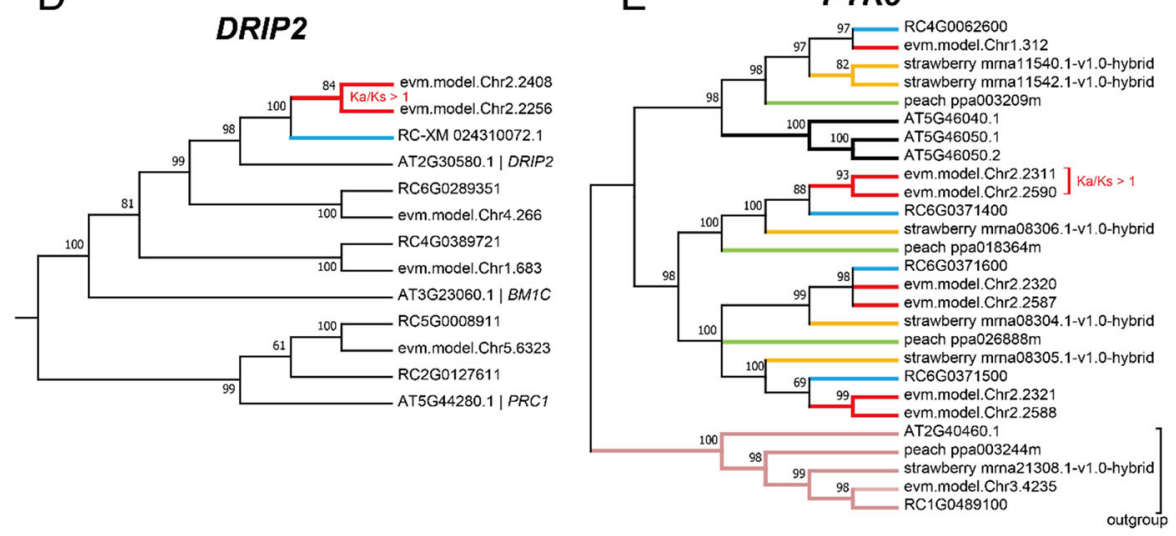

Fig. 6 Abiotic stress-related genes were enriched in Rosa rugosa. A The agriGO modules of salt stress- and water stress-related genes predicted using Arabidopsis orthologs of R. rugosa duplicated genes after WGT. B, C Venn clustering of 193 and 103 abiotic stress-related genes from R. rugosa and $R$. chinensis, respectively. D, E The DRIP2 genes have two paralogs in $R$. rugosa but one in $R$. chinensis and Arabidopsis. The PTR3 genes have 7 paralogs in $R$. rugosa but 4 in $R$. chinensis. The DRIP2 paralogs and two PTR3 paralogs in $R$. rugosa have been subjected to strong positive selection pressure 


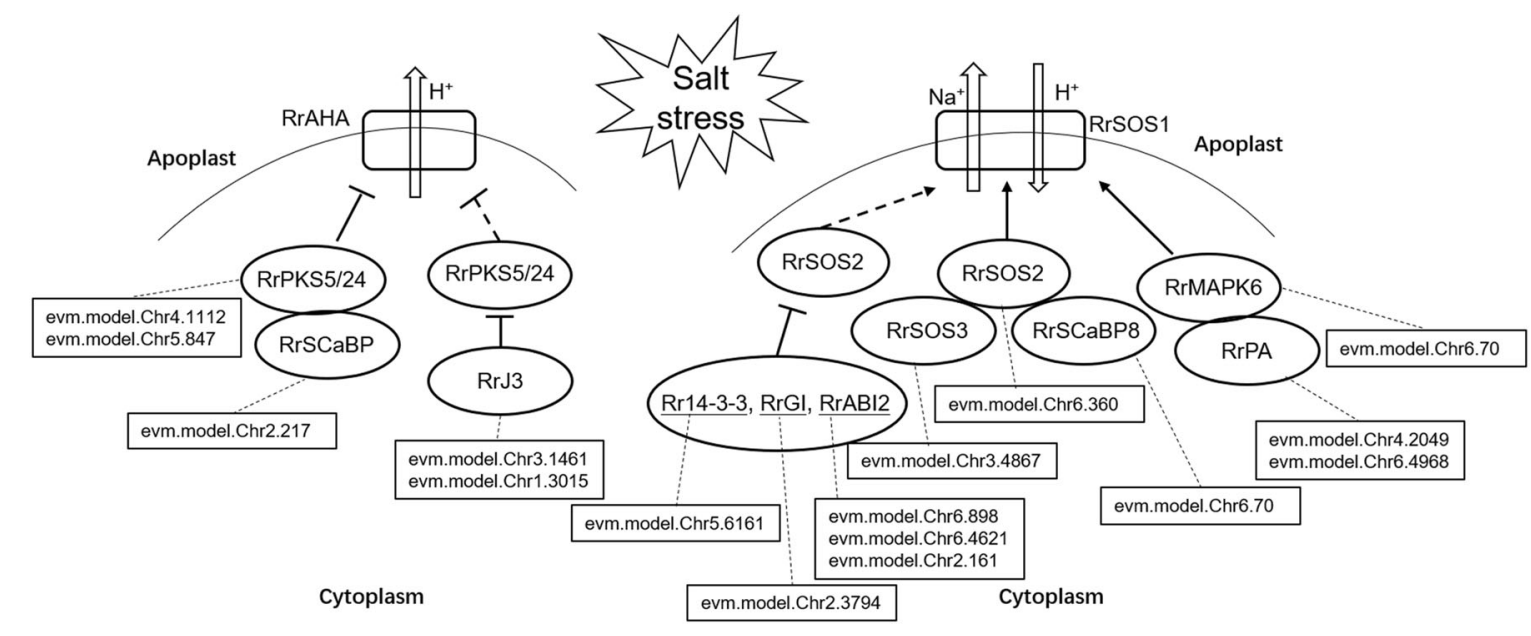

Fig. 7 The deduced salt stress response signaling pathway in $\boldsymbol{R}$. rugosa. The related $R$. rugosa genes were listed in rectangular boxes

Then, Juicer was used to filter the sequence and cluster it, and the Juicerbox tool ${ }^{30}$ was applied to manually adjust chromosome construction. We finally anchored the scaffolds on seven chromosomes. In addition, the BUSCO v3.0.2 $2^{31}$ pipeline was used to assess the completeness and accuracy of the $R$. rugosa genome with the embryophyte_odb10 dataset, which contains 1614 BUSCO gene sets.

\section{Genome annotation}

To annotate the repeat sequence in $R$. rugosa, RepeatModeler v2.0.1 ${ }^{32}$ and RepeatMasker v4.1.0 ${ }^{33}$ were searched using Repbase TE libarary (v2018.10.26) from the Repbase server (https://www.girinst.org/repbase/) ${ }^{34}$. To predict the protein-coding gene $R$. rugosa, we combined de novo gene prediction, homology-based prediction and RNA-seq-based prediction. SNAP v2006.07.28 ${ }^{35}$ and AUGUSTUS v3.3.3 ${ }^{36}$ were used for de novo prediction with the parameter file trained on $F$. vesca, M. domestica, P. persica, P. bretschneideri, R. chinensis and R. occidentalis. For homology-based and RNA-seq-based gene identification, $F$. vesca, $M$. domestica, $P$. persica, $P$. bretschneideri, $R$. chinensis and $R$. occidentalis genomes were searched. Then, we mapped RNA-seq data to the genome by Hisat 2 v2.2.1 ${ }^{37}$ and obtained gene models with SAMtools v1.7.1 $1^{38}$. These transcripts and the genes from the six homologous species were analyzed with GeMoMa v1.6.4 software to identify protein-coding genes ${ }^{39}$. Finally, we merged the gene models with EVidenceModeler V1.1.1 ${ }^{40}$ from SNAP v2006.07.28, AUGUSTUS V3.3.3 and GeMoMa v1.6.4. We annotated the COG/KOG ${ }^{41}$, Gene Ontology $^{42}$ and KEGG pathways ${ }^{43}$ of rosa protein sequences on the eggNOG-mapper online website (http:// eggnog-mapper.embl.de/) and used HMMER v3.3.1 ${ }^{44}$ with the Pfam database ${ }^{45}$ to identify the functions of all proteins.

\section{Construction of phylogenetic trees and estimation of divergence times}

We used OrthoFinder v2.4.0 ${ }^{46}$ to generate clusters of gene families from rugged rose ( $R$. rugosa), Arabidopsis (A. thaliana), strawberry ( $F$. vesca), $M$. domestica, $P$. persica, $P$. bretschneideri, $R$. chinensis and $V$. vinifera. We aligned the single-copy proteins generated from OrthoFinder v2.4.0 ${ }^{46}$ by MUSCLE v3.8.1551 ${ }^{47}$. Based on the single-copy nuclear genes from the MUSCLE results, we used RAxML v8.2.12 ${ }^{48}$ and ASTRAL-II v5.7.3 ${ }^{49}$ to construct the phylogenetic tree with the maximum-likelihood method. Then, we used the MCMCTree pipeline of the PAML v $4.9^{50}$ program to calculate the divergence times of the eight species. We marked the split times of Rosids and Rosaceae that were downloaded from the TimeTree website (http://timetree.org/).

\section{Gene family expansion and contraction}

Based on the gene family and gene count statistics of OrthoFinder v2.4.0, we used CAFÉ v4.2.1 ${ }^{51}$ to determine the expansion and contraction gene families of $R$. rugosa, $A$. thaliana, F. vesca, M. domestica, P. persica, P. bretschneideri, $R$. chinensis and $V$. vinifera with a $p$ value $<0.01$.

\section{Synteny and WGD}

To find the synteny blocks between $R$. rugosa, $R$. chinensis and $V$. vinifera, the python version of MCScan (JCVI v1.1.7) ${ }^{52}$ was applied to compare proteins to proteins. We set 30 genes as the minimum in a syntenic region. Furthermore, we constructed a Circos map by Circos v0.52 $2^{53}$. To analyze whole-genome duplications in 
rosa, we calculated and mapped the $K \mathrm{~s}$ values and distribution by wgd v1.1.0 $0^{54}$.

\section{Genes under positive selection}

To analyze positively selected genes, we chose $F$. vesca, M. domestica, P. persica, P. bretschneideri and $R$. chinensis to identify orthologs by WGD. ParaAT v2.0 $0^{55}$ and KaKs_Calculator v2.0 ${ }^{56}$ were used to detect the genes under positive selection. Next, we used BLASTP to search for homologous genes between $R$. rugosa and A. thaliana. AgriGO v2.0 ${ }^{57}$ was used to annotate the GO, and we drew a Venn diagram on an online website (http:// bioinformatics.psb.ugent.be/webtools/Venn/).

\section{Acknowledgements}

F.C. acknowledges funding from the National Natural Science Foundation of China (31801898). This work is supported by the high-performance computing platform of the Bioinformatics Center, Nanjing Agricultural University. This work is supported by the Fundamental Funds for the Central Universities, NJAU (KYXJ202004). We thank the Priority Academic Program Development of Jiangsu Higher Education Institutions for funding.

\section{Author details \\ ${ }^{1}$ College of Horticulture, Nanjing Agricultural University, Nanjing 210095, China. ${ }^{2}$ Academy for Advanced Interdisciplinary Studies, Nanjing Agricultural University, Nanjing 210095, China. ${ }^{3}$ BGl-Shenzhen, Beishan Industrial Zone, Yantian District, Shenzhen 518083, China. ${ }^{4}$ Department of Biology, University of Copenhagen, Copenhagen, Denmark. ${ }^{5}$ Grandomics Biosciences Co., Ltd, Wuhan, China. ${ }^{6}$ School of Life Sciences, Nanjing University, Nanjing, China. ${ }^{7}$ College of life science, Nantong University, Nantong, China. ${ }^{8}$ Genomics and Genetic Engineering Laboratory of Ornamental Plants, College of Agriculture and Biotechnology, Zhejiang University, Hangzhou, China. ${ }^{9}$ Department of plant sciences, University of Tennessee, Knoxville, TN, USA}

\section{Author contributions}

Z.-M.(Max)C. and F.C. designed and led the project. F.C. and L.S. carried out the genomic analyses and wrote the draft manuscript. S.H., J.X., G.L., H.L., Y.J., J.D., Y. Q., Y.F., H.L., Q.Y., W.L., Z.S., J.Z., L.Z., F.C. participated in genomic assembly, annotation, and analyses. All the authors approved the final manuscript.

\section{Data availability}

All the raw data, as well as genome sequences, protein sequences, CDSs, and GFF files, could be found in our eplant database (http://eplant.njau.edu.cn).

\section{Conflict of interest}

The authors declare no competing interests.

Supplementary information The online version contains supplementary material available at https://doi.org/10.1038/s41438-021-00594-z.

Received: 18 March 2021 Revised: 12 April 2021 Accepted: 11 May 2021 Published online: 18 June 2021

\section{References}

1. Altiner, D. \& Kilicgun, H. The antioxidant effect of Rosa rugosa. Drug Metabol. Drug Interact. 23, 323-327 (2008).

2. Cendrowski, A., Krasniewska, K, Przybyl, J.L., Zielinska, A. \& Kalisz, S. Antibacterial and antioxidant activity of extracts from rose fruits (Rosa rugosa). Molecules $\mathbf{2 5}$, 1365 (2020).

3. Belcher, C. Effect of sand cover on the survival and vigor of Rosa rugosa. Int. J. Biometeorol. 21, 276-280 (1977).

4. Hashidoko, Y. The phytochemistry of Rosa rugosa. Phytochemistry 43, 535-549 (1996).
5. Stefanowicz, A. M. et al. Invasion of Rosa rugosa induced changes in soil nutrients and microbial communities of coastal sand dunes. Sci. Total Environ 677, 340-349 (2019).

6. Chen, F. et al. The sequenced angiosperm genomes and genome databases. Front. Plant Sci. 9, 418 (2018).

7. Zhang, L. et al. The water lily genome and the early evolution of flowering plants. Nature 577, 79-84 (2020).

8. Chen, F. et al. Genome sequences of horticultural plants: past, present, and future. Hortic. Res. 6, 112 (2019).

9. Wissemann, V. \& Ritz, C. M. The genus Rosa (Rosoideae, Rosaceae) revisited: molecular analysis of nrlTS-1 and atpB-rbcL intergenic spacer (IGS) versus conventional taxonomy. Bot. J. Linn. Soc. 147, 275-290 (2005).

10. Nakamura, N. et al. Genome structure of Rosa multiflora, a wild ancestor of cultivated roses. DNA Res. 25, 113-121 (2018).

11. Raymond, O. et al. The Rosa genome provides new insights into the domestication of modern roses. Nat. Genet. 50, 772-777 (2018).

12. Hibrand Saint-Oyant, L. et al. A high-quality genome sequence of Rosa chinensis to elucidate ornamental traits. Nat. Plants 4, 473-484 (2018).

13. Kim, Y. et al. The complete chloroplast genome of candidate new species from Rosa rugosa in Korea (Rosaceae). Mitochondrial DNA B Resour. 4 2433-2435 (2019).

14. Park, J., Xi, H., Kim, Y., Nam, S. \& Heo, K. I. The complete mitochondrial genome of new species candidate of Rosa rugosa (Rosaceae). Mitochondrial DNA B Resour. 5, 3435-3437 (2020).

15. Edger, P. P. et al. Single-molecule sequencing and optical mapping yields an improved genome of woodland strawberry (Fragaria vesca) with chromosome-scale contiguity. Gigascience 7, 1-7 (2018).

16. Shulaev, V. et al. The genome of woodland strawberry (Fragaria vesca). Nat. Genet. 43, 109-116 (2011).

17. Zhang, J. et al. The high-quality genome of diploid strawberry (Fragaria nilgerrensis) provides new insights into anthocyanin accumulation. Plant Biotechnol. J. 18, 1908-1924 (2020).

18. Liu, C. Y. et al. Phylogenetic Relationships in the genus Rosa revisited based on rpl16, trnL-F, and atpB-rbcL sequences. Hortscience 50, 1618-1624 (2015).

19. Zhang, L. et al. The ancient wave of polyploidization events in flowering plants and their facilitated adaptation to environmental stress. Plant Cell Environ. $\mathbf{4 3}$, 2847-2856 (2020)

20. Van de Peer, Y., Mizrachi, E. \& Marchal, K. The evolutionary significance of polyploidy. Nat. Rev. Genet. 18, 411-424 (2017).

21. Jaillon, $\mathrm{O}$. et al. The grapevine genome sequence suggests ancestral hexaploidization in major angiosperm phyla. Nature 449, 463-467 (2007).

22. Xiang, Y. et al. Evolution of Rosaceae fruit types based on nuclear phylogeny in the context of geological times and genome duplication. Mol. Biol. Evol. 34, 262-281 (2017)

23. Zhou, W. et al. Studies of aroma components on essential oil of Chinese kushui rose. Se Pu 20, 560-564 (2002).

24. Feng, L. et al. Flowery odor formation revealed by differential expression of monoterpene biosynthetic genes and monoterpene accumulation in rose (Rosa rugosa Thunb.). Plant Physiol. Biochem. 75, 80-88 (2014).

25. Chen, F., Tholl, D., Bohlmann, J. \& Pichersky, E. The family of terpene synthases in plants: a mid-size family of genes for specialized metabolism that is highly diversified throughout the kingdom. Plant J. 66, 212-229 (2011).

26. Nurk, S. et al. HiCanu: accurate assembly of segmental duplications, satellites, and allelic variants from high-fidelity long reads. Genome Res. 30, 1291-1305 (2020).

27. Pryszcz, L. P. \& Gabaldon, T. Redundans: an assembly pipeline for highly heterozygous genomes. Nucleic Acids Res. 44, e113 (2016).

28. Li, H. \& Durbin, R. Fast and accurate short read alignment with BurrowsWheeler transform. Bioinformatics 25, 1754-1760 (2009).

29. Dudchenko, O. et al. De novo assembly of the Aedes aegypti genome using $\mathrm{Hi}-\mathrm{C}$ yields chromosome-length scaffolds. Science 356, 92-95 (2017).

30. Durand, N. C. et al. Juicebox provides a visualization system for Hi-C contact maps with unlimited zoom. Cell Syst. 3, 99-101 (2016).

31. Seppey, M., Manni, M. \& Zdobnov, E. M. BUSCO: assessing genome assembly and annotation completeness. Methods Mol. Biol. 1962, 227-245 (2019).

32. Flynn, J. M. et al. RepeatModeler2 for automated genomic discovery of transposable element families. Proc. Natl Acad. Sci. USA. 117, 9451-9457 (2020).

33. Tempel, S. Using and understanding RepeatMasker. Methods Mol. Biol. 859, 29-51 (2012)

34. Bao, W., Kojima, K. K. \& Kohany, O. Repbase Update, a database of repetitive elements in eukaryotic genomes. Mob. DNA 6, 11 (2015). 
35. Korf, I. Gene finding in novel genomes. BMC Bioinforma. 5, 59 (2004).

36. Stanke, M., Schoffmann, O., Morgenstern, B. \& Waack, S. Gene prediction in eukaryotes with a generalized hidden Markov model that uses hints from external sources. BMC Bioinforma. 7, 62 (2006).

37. Kim, D., Langmead, B. \& Salzberg, S. L. HISAT: a fast spliced aligner with low memory requirements. Nat. Methods 12, 357-360 (2015).

38. Li, H. et al. Genome Project Data Processing S: The Sequence Alignment/Map format and SAMtools. Bioinformatics 25, 2078-2079 (2009).

39. Keilwagen, J., Hartung, F. \& Grau, J. GeMoMa: homology-based gene prediction utilizing intron position conservation and RNA-seq data. Methods Mol. Biol. 1962, 161-177 (2019).

40. Haas, B. J. et al. Automated eukaryotic gene structure annotation using EVidenceModeler and the program to assemble spliced alignments. Genome Biol. 9, R7 (2008).

41. Tatusov, R. L., Galperin, M. Y., Natale, D. A. \& Koonin, E. V. The COG database: a tool for genome-scale analysis of protein functions and evolution. Nucleic Acids Res. 28, 33-36 (2000).

42. Ashburner, M. et al. Gene ontology: tool for the unification of biology. The Gene Ontology Consortium. Nat. Genet. 25, 25-29 (2000).

43. Kanehisa, M. \& Goto, S. KEGG: kyoto encyclopedia of genes and genomes. Nucleic Acids Res. 28, 27-30 (2000).

44. Johnson, L. S., Eddy, S. R. \& Portugaly, E. Hidden Markov model speed heuristic and iterative HMM search procedure. BMC Bioinforma. 11, 431 (2010).

45. Finn, R. D. et al. Pfam: the protein families database. Nucleic Acids Res. 42 D222-D230 (2014). Database issue.

46. Emms, D. M. \& Kelly, S. OrthoFinder: phylogenetic orthology inference for comparative genomics. Genome Biol. 20, 238 (2019).
47. Edgar, R. C. MUSCLE: multiple sequence alignment with high accuracy and high throughput. Nucleic Acids Res. 32, 1792-1797 (2004).

48. Stamatakis, A. RAxML version 8: a tool for phylogenetic analysis and postanalysis of large phylogenies. Bioinformatics 30, 1312-1313 (2014).

49. Mirarab, S. \& Warnow, T. ASTRAL-II: coalescent-based species tree estimation with many hundreds of taxa and thousands of genes. Bioinformatics 31, i44-i52 (2015)

50. Yang, Z. PAML 4: phylogenetic analysis by maximum likelihood. Mol. Biol. Evol. 24, 1586-1591 (2007).

51. De Bie, T., Cristianini, N., Demuth, J. P. \& Hahn, M. W. CAFE: a computational tool for the study of gene family evolution. Bioinformatics 22, 1269-1271 (2006).

52. Tang, $\mathbf{H}$. et al. Synteny and collinearity in plant genomes. Science $\mathbf{3 2 0} \mathbf{4} \mathbf{4 8 6}-\mathbf{4 8 8}$ (2008).

53. Krzywinski, M. et al. Circos: an information aesthetic for comparative genomics. Genome Res. 19, 1639-1645 (2009).

54. Zwaenepoel, A. \& Van de Peer, Y. wgd-simple command line tools for the analysis of ancient whole-genome duplications. Bioinformatics 35, 2153-2155 (2019).

55. Zhang, Z. et al. ParaAT: a parallel tool for constructing multiple proteincoding DNA alignments. Biochem. Biophys. Res. Commun. 419, 779-781 (2012).

56. Wang, D., Zhang, Y., Zhang, Z., Zhu, J. \& Yu, J. KaKs_Calculator 2.0: a toolkit incorporating gamma-series methods and sliding window strategies. Genomics Proteom. Bioinforma. 8, 77-80 (2010).

57. Tian, T. et al. agriGO v2.0: a GO analysis toolkit for the agricultural community, 2017 update. Nucleic Acids Res. 45, W122-W129 (2017). 